\title{
The Price of ANARChy of A Network
} CREATION GAME WITH EXPONENTIAL PAYOFF by

\author{
NADINE BAUMANN \\ Sebastian S TILleR
}

No. $2007 / 19$ 


\title{
The Price of Anarchy of a Network Creation Game with Exponential Payoff
}

\author{
Nadine Baumann* Sebastian Stiller ${ }^{\dagger}$
}

May 8, 2007

\begin{abstract}
We analyze a graph process (or network creation game) where the vertices as players can establish mutual relations between each other at a fixed price. Each vertex receives income from every other vertex, exponentially decreasing with their distance. To establish an edge, both players have to make a consent.

We show that the process has a positive probability to cycle. We reduce the creation rule with payoff functions to graph theoretic criteria. Moreover, these criteria can be evaluated locally. This allows us to thoroughly reveal the structure of all stable states. In addition, the question for the price of anarchy can be reduced to counting the maximum number of edges of a stable graph. This together with a probabilistic argument allows to determine the price of anarchy precisely.
\end{abstract}

*Universität Dortmund, Fachbereich Mathematik, 44221 Dortmund, Germany.

Email: nadine.baumann@math. uni-dortmund.de.

Work supported by DFG Focus Program 1126, "Algorithmic Aspects of Large and Complex Networks", grant no. SK 58/4-1 and SK 58/5-3.

${ }^{\dagger}$ Technische Universität Berlin, Fakultät II, Straße des 17. Juni 136, 10623 Berlin, Germany.

Email: stiller@math.tu-berlin.de.

Work supported by the ARRIVAL project, within the 6th Framework Programme of the European Comission under contract no. FP6-021235-2 


\section{Introduction}

A fundamental Graph Process. Graph processes and network creation games help to understand the structure of real-world networks. Though these tools often fall short of a detailed modeling, their analysis elates by linking a simple and intuitive, creative principle to typical features of huge real-world networks. In this way, e.g., the preferential attachment model (see Barabási and Albert [6] for details) explains the scale-free structure formed by the pages of the WWW and their links.

Several such models have been proposed particularly in an economic context. We consider one which seems fundamental among these. The network created is a simple, undirected graph $G(V, E)$. It is created step by step. In each step a pair of vertices $\{u, v\}$ is chosen with no respect to whether their common edge already exists or not. For the edge to exist at the end of the step both vertices $u$ and $v$ have to benefit from it. In case at least one of them disapproves, the edge will not be present at the end of the step. A vertex benefits from an edge $e$, if the current graph with $e$ gives that vertex a higher payoff than the current graph without $e$. The costs are local, namely every vertex pays a factor $c$ times its degree, but the vertex enjoys income globally, namely from every other vertex exponentially declining with the distance to the other vertex. Whether to be at distance 2 or 3 is a much greater difference than whether to be at distance 100 or 101. These intuitions are not limited to economics. Think of the network as a means to send information from any vertex to any other vertex along paths. Unfortunately, at every edge used a $(1-\delta)$-portion of the information sent into the edge is lost. (Equivalently, each edge can have a probability of temporary failure of $(1-\delta)$.) Hence, every vertex $v$ will send its deliveries to $w$ via a shortest path and only a portion of $\delta^{\operatorname{dist}(v, w)}$ of the total amount sent from $v$ to $w$ and vice versa will reach its destination.

The creation rule can be understood as a game, where the vertices as players create the edges myopicly, selfishly, and uncooperatively, though each edge requires both its end-vertices to agree. Obviously, it depends on the order of steps which networks are created. One is interested in certain equilibria or stable states, i.e., situations which every player would leave unchanged, if it was her turn now. The notion of stability suitable to this model is called pairwise stability (cf. Jackson [11]), i.e., a graph is stable, if it will stay unchanged, no matter which pair of vertices is chosen for the next step. Alternatively, one can define a probability distribution according to which the next pair of players is chosen. Then the game becomes a random graph process, i.e., a sequence of random variables $\left(G_{i}\right)_{i \in \mathbb{N}}$, each one representing a network. Again the same stable states and the possibility for the process to cycle are of primary interest. For the graph process, we accept every distribution that assigns a positive probability to every pair of vertices.

Besides the stable graphs, one is interested in graphs maximizing the sum of the payoff, i.e., the total throughput of information minus the total edge costs. These graphs are called efficient graphs or system optima. The smallest ratio between the total payoff of a stable graph and that of a system optimum is called the price of anarchy of the graph process and is of high interest in network creation games since its first mentioning by Koutsoupias and Papadimitriou [13]. The classical notion of Nash-equilibrium (cf. [15]) is not adequate for bilateral games. Note that pairwise stability is a stricter notion of equilibrium as a player is not allowed to overhaul his whole strategy without the other players reacting to his steps (cf. also [7]).

Related Results. The huge number of network creation games proposed in the literature shows the great interest for these explanatory tools. See Jackson [11] for a survey article. During the previous decade, also the interest in the analysis especially of the price of anarchy and Nash equilibria of those network creation games increased. The first to treat the price of anarchy were Koutsoupias and Papadimitriou in their seminal paper [13]. A relevant but simple network creation game is the unilateral game with linear payoff function as proposed by Fabrikant et al. [9]. Their model is the same as ours except for two features: For them an edge is used mutually, but only one of its end-vertices pays for it. Second, the income from other vertices decreases linearly with their distance. Recently, Albers et al. [1] give the best known upper bound on the price of anarchy for this model. Moreover, they disprove a structural conjecture for stable states made by 
Fabrikant et al. [9] when they show that graphs with cycles can be stable.

Corbo and Parkes [7] analyzed a bilateral consent-driven variant of the model by Fabrikant et al. and determined lower and upper bounds of the price of anarchy. Further, they compared these values with the unilateral model. As already stated by Corbo and Parkes, Nash equilibria cannot be determined for bilateral games. They therefore introduced the alleviated notion of pairwise Nash which is equivalent pairwise stability.

The model we consider uses a more elaborate payoff function and the bilateral approach for sharing the costs of the edge. It was proposed by Jackson and Wolinsky [12] and interpreted as a graph process by Watts [16]. We state their results in Section 2. Though in comparison to those of [9] and [1] as well as [7] their results may appear limited to peculiar cases and immediate from the definition, the model of [12] and [16] is more convincing for two reasons: They give a consistent interpretation of mutual relations. And the income decreases exponentially with the distance.

Another model using bilateral cost sharing is given by Melendez-Jiminez [14]. Models using cost sharing principles are for example Bala and Goyal [5]. Anshelevich et al. [4] and [3] establish a near optimal solution for selfish players and determine the price of anarchy in a model with fair costs.

Our Contribution. It would be desirable to achieve the same level of mathematical insight for the process of Jackson, Wolinsky [12] and Watts [16] as provided for the process of Fabrikant et al. [9] by Albers et al. [1], namely some structural knowledge of the stable states and bounds on the price of anarchy. We achieve even more. Our process depends on two parameters, $c$ and $\delta$. First, we show that this process behaves equally whenever $c$ is in $\left(\delta-\delta^{2}, \delta-\delta^{3}\right)$. For these cases we show that the process has positive probability to cycle.

Further, we provide for thorough structural insight to stable states. On the one hand, this is of interest in its own. On the other hand, it allows for our main theorem: We give an explicit formula in $c, \delta$, and the number of vertices for the exact price of anarchy for all $c \in\left(\delta-\delta^{2}, \delta-\delta^{3}\right)$. We argue by reducing the creation rule and payoff functions to local, graph theoretic criteria. In particular, the price of anarchy can be reduced to the number of edges in a maximum stable graph.

For $c>\delta-\delta^{3}$ we indicate how and to which extend our methods can be carried over. Further, we show how an analogon to our main result is linked to extremal graph theory.

\section{Preliminaries}

For a graph $G$ as usual $V(G)$ and $E(G)$ stand for its vertex and its edge set. For a pair of vertices $e=\{u, v\}$, we use $G+e$ and $G-e$, no matter whether $e \in E(G)$ or not, to denote the graph $G$ with or without the edge $e$. The neighborhood of a vertex $v$ will be symbolized by $N(v)$, its degree by $d(v)(=|N(v)|)$ and the distance between two vertices $u$ and $v$ by $\operatorname{dist}(u, v)$, i.e., the minimum number of edges of a path connecting $u$ and $v$. If there does not exist a path, we say $\operatorname{dist}(u, v)=\infty$.

Formally we define a graph process (or a game) to be a triple of the cost coefficient, the income basis and the number of vertices, $(c, \delta, n) \in \mathbb{R} \times(0,1) \times \mathbb{N}$.

For every vertex $v$ the income is a function of $E(G)$ given by $\sum_{u \in V \backslash\{v\}} \delta^{\operatorname{dist}(u, v)}$. The costs of a vertex $v$ are $c \cdot d(v)$, and its payoff is its income minus its costs. The total (or social) payoff is the sum of all vertices' payoff: $\sum_{v \in V}\left(\sum_{u \in V \backslash\{v\}} \delta^{\operatorname{dist}(u, v)}-c \cdot d(v)\right)$. A graph maximizing the total payoff is called system optimum.

A situation is a graph $G$ together with a pair $\{u, v\}$ of its vertices. Every situation defines a polynomial in $\delta$ for each of the pair's vertices $u$ and $v$, expressing the change in income (not yet in payoff) for that vertex between $G-e$ and $G+e$, with $e=\{u, v\}$. In these terms the creation rule reads as follows: When the (possible) edge $e=\{u, v\}$ is evaluated given the graph $G$, the decision will be positive, i.e., $G+e$ will be 
the resulting graph, if both polynomials are bigger than $c$, and negative if at least one is smaller, i.e., $G-e$ results. We do not consider cases where a polynomial can equal $c$, i.e., a vertex is indifferent about an edge. It would be possible to extend the model and results to these cases, but it would also be tedious.

Observe that if an edge is inserted by the process, this can only increase the total payoff. The deletion of an edge can be locally advantageous but decrease the total payoff.

A graph $G$ is called stable, if $G$ together with any $e=\{u, v\} \in E(G)$ is a situation with positive decision, and $G$ together with any $e=\{u, v\} \notin E(G)$ is a situation with negative decision. The price of

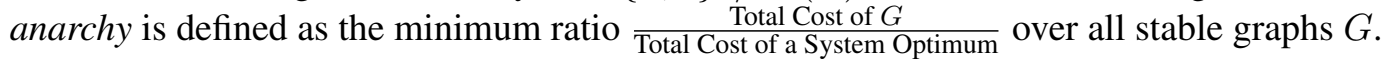

Expressed in these terms Jackson and Wolinsky [12] and Watts [16] observe that for $c<\delta-\delta^{2}$ the complete graph is the only stable graph and the unique system optimum, because, no matter what the graph looks like, every further edge is beneficial. Trees have the least total cost among all connected graphs. In a star all not directly connected pairs of vertices are at distance 2 . Therefore, if the cost factor $c$ is high enough to draw any attention to the costs the star is optimal, namely for $c \in\left(\delta-\delta^{2}, \delta+\frac{n-2}{2} \delta^{2}\right)$. Beyond that limit for the costs, even the star's payoff becomes negative and the empty graph is the system optimum. Notably, the star is a stable graph for $c \in\left(\delta-\delta^{2}, \delta\right)$. Beyond that the empty graph is a stable state (though not the only one).

A result which concludes from the stability and the chosen values of $c$ and $\delta$ to structural properties of the graph yields our first and obvious lemma.

Lemma 1. If a graph $G$ is a stable state of a graph process with $c<\delta-\delta^{k+1}, k \in \mathbb{N}$, then $G$ has diameter less than or equal to $k$.

Proof. Assume to the contrary that there are two vertices $u$ and $v$ at distance greater than $k$. The (nonexisting) edge $\{u, v\}$ would at least improve the income for $u$ from $v$, i.e., the increase in income is greater than or equal to $\delta-\delta^{k+1}$. As the analogon holds for $v$, the edge would be inserted, which is to say, the graph is unstable.

\section{The Graph Process for $c \in\left(\delta-\delta^{2}, \delta-\delta^{3}\right)$}

In this main section we restrict to the case $c \in\left(\delta-\delta^{2}, \delta-\delta^{3}\right)$. The restriction has the nice property that all graph processes in those cases are identical. We say that a set of graph processes $\{(c, \delta, n)\}$ is identical, if and only if for every situation the decision is the same for all processes in the set.

Theorem 1. For fixed $n$ the set of graph processes $\left\{(c, \delta, n) \mid c \in\left(\delta-\delta^{2}, \delta-\delta^{3}\right)\right\}$ is identical.

Proof. Let $G$ be the graph and $e=\{u, v\}$ be the edge of an arbitrary situation on $n$ vertices. If $\operatorname{dist}_{G-e}(u, v)$ in the current graph without $e$ is greater than or equal to 3 , the increase in the income is for each vertex at least $\delta-\delta^{3}$, because this is the minimal improvement in the income from $u$ for $v$ and vice versa. Further vertices could only lead to a bigger increase, as no distance gets longer by inserting an edge. As $c<\delta-\delta^{3}$, the increase in income is strictly greater than that of the costs, and the payoff is positive.

Assume now $\operatorname{dist}_{G-e}(u, v)=2$ for the situation considered, and let $\delta$ and $c$ be parameters such that the decision is in favor of $e$. Then the gain for $v$ from $u$ (and vice versa) is only $\delta-\delta^{2}$, which is less than the cost of an extra edge. This requires for both $u$ and $v$ that further vertices are closer in $G+e$ than in $G-e$. For some $x \neq v$ any shortest path from $u$ to $x$ in $G+e$ must use the edge $e$, i.e., is of the form: $(u, v, \ldots, x)$. This implies, that at least for one neighbor $y$ of $v$ all shortest path from $u$ to $y$ in $G+e$ go via $v$ (and are shorter than those in $G-e$ ). We can conclude that the change in income for $u$ from $y$ is at least $\delta^{2}-\delta^{3}$, as $\operatorname{dist}_{G-e}(u, y) \geq 3$. Therefore, the total change in income for $u$ is at least the sum of the changes stemming from $v$ and $y$, which is at least $\delta-\delta^{2}+\delta^{2}-\delta^{3}=\delta-\delta^{3}$. An analogon holds for $v$. As this is the case, the situation yields a positive decision for all $c$ and $\delta$ that meet $c<\delta-\delta^{3}$. 


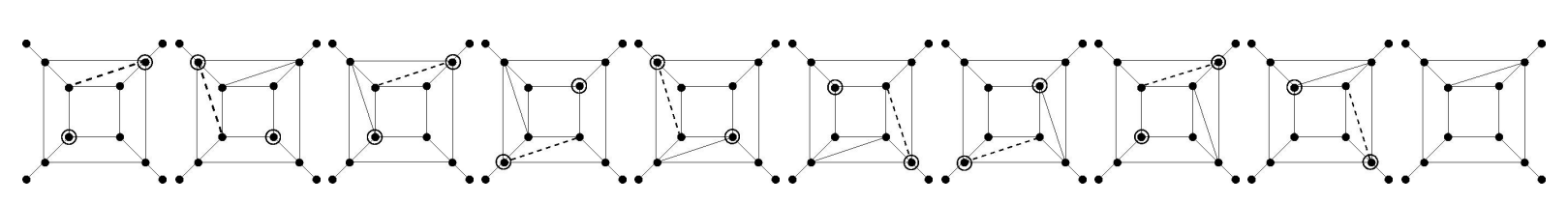

Figure 1: Sequence of the graph process where edges are inserted and deleted (to be read from the left to the right). The edge in question is the dashed one.

Altogether, for a given situation the decision will either be positive for all graph processes with $c \in$ $\left(\delta-\delta^{2}, \delta-\delta^{3}\right)$ or negative for all of them.

The argument of the theorem allows for all graph processes with $c \in\left(\delta-\delta^{2}, \delta-\delta^{3}\right)$ to reduce a decision to a graph theoretical set of rules. An edge $e=\{u, v\}$ will be kept or inserted, if and only if at least one of the following conditions holds in the graph without $e$.

1. We have $\operatorname{dist}_{G-e}(u, v)>2$.

2. The end-vertex $u$ has a neighbor $x$ with $\operatorname{dist}_{G-e}(v, x)=3$, and the end-vertex $v$ has a neighbor $y$ with $\operatorname{dist}_{G-e}(u, y)=3$.

As all graph processes on $n$ vertices are identical under the restriction $c \in\left(\delta-\delta^{2}, \delta-\delta^{3}\right)$, we also speak of the graph process.

\subsection{Cycling}

Since the notion of stability of a graph process is important for the determination of the price of anarchy, we also have to give an idea whether the process always terminates in a stable graph. The following theorem shows that there is an infinite series of pairs of vertices that never lead to a stable graph.

Theorem 2. The graph process for parameters $c$ and $\delta$ with $c \in\left(\delta-\delta^{2}, \delta-\delta^{3}\right)$ can cycle.

Proof. The first graph in Figure 1 (without the dashed edge) may occur, as it contains no triangles. By the choice of $c$ an $\delta$, every vertex seeks to be connected to every other vertex with distance less than 3 . The vertices of the inner square are always interested in edges to the vertices of the outer square, because the latter have exclusive vertices. The interest of the outer square's vertex is decisive for an edge to exist. Consider Figure 1 which depicts a possible sequence of the graph process where the edge in question is always the dashed one. It turns out, that the edges chosen by the graph process already in the graph will be deleted and edges not in the graph will be inserted during this step of the graph process. The dashed edge in the first picture in Figure 1 is inserted to provide for a distance of 2 between the vertices marked with a circle. The same happens for the edge in question in the next step of the process, depicted in the second picture. In the third picture, the edge chosen by the graph process is already in the graph. The two marked vertices are currently connected by two paths of length 2 . As one of the vertices is an end-vertex of the edge in question, the edge is therefore removed. The remaining pictures show how this procedures leads back to the initital graph (last picture). Thus, the process cycles for the given values of $c$ and $\delta$.

\subsection{The Price of Anarchy}

In order to determine the price of anarchy, we need to establish criterions for stable graphs for the considered graph process. A first result follows immediately from Lemma 1 which amounts to say that every stable graph must have diameter exactly 2 , as the complete graph obviously is not stable.

Corollary 1. The star is the only stable tree. 
Moreover, we can give a broader sufficient condition for a graph to be stable.

Theorem 3. A graph $G$ is stable, if $G$ has diameter 2 and contains no triangles.

Proof. For stability, on the one hand, we have to show that no edge in $G$ will be removed. The graph $G$ contains no triangles. Hence the shortest path between the end-vertices $u$ and $v$ of a currently present edge $e$ in the graph without that edge, $G-e$, has length greater than or equal to 3 . Thus, by the same argument as in Lemma 1, the edge is beneficial for both its endpoints and therefore kept.

On the other hand, no further edge will be inserted as the diameter suitable to the parameters is already reached: Choose an edge $e=\{u, v\}$ that is not present in $G$ and for vertex $u$ define $A_{G}:=$ $\{x \in V(G) \mid \operatorname{dist}(u, x)=1\}, B_{G}:=\{x \in V(G) \mid \operatorname{dist}(u, x)=2\}$ and $A_{G+e}, B_{G+e}$ respectively. Then $A_{\bullet} \cup B_{\bullet} \cup\{u\}=V$ for both graphs, and $A_{G} \cup\{v\}=A_{G+e}$ and $B_{G+e} \cup\{v\}=B_{G}$, where all unions are disjoint. Consequently, the change in income by inserting $e$ is exactly $\delta-\delta^{2}$ and therefore it is not beneficial to insert $e$. Since this argument holds for all choices of $e$ and $u$, the graph is stable.

By the above observations, we reduced the decisions of any situation to graph theoretic considerations. In fact, we can do the same for the price of anarchy, or equivalently, the total cost of a stable graph. For a stable graph we know the diameter to be less than or equal to 2 . Consequently, we can rewrite the total benefit of such a graph in the following simple way.

$$
\sum_{u \in V}\left(\sum_{v \in V \backslash\{u\}} \delta^{\operatorname{dist}(u, v)}-d(u) c\right)=\sum_{\{u, v\} \in E}(\delta-c)+\sum_{\substack{\{u, v\} \in E, \operatorname{dist}(u, v)=2}} \delta^{2}=m(\delta-c)+\left(\left(\begin{array}{c}
n \\
2
\end{array}\right)-m\right) \delta^{2}
$$

Here $m$ denotes the number of edges. As $\delta-c-\delta^{2}<0$ by the choice of the parameters, it directly follows that the benefit of a stable graph is the bigger the less edges it has.

Theorem 4. Let $G$ and $G^{\prime}$ be stable graphs. The total payoff of $G$ is greater than that of $G^{\prime}$ if and only if $|E(G)|<\left|E\left(G^{\prime}\right)\right|$.

This together with the description of a stable graph in Theorem 3 provides for a lower bound on the price of anarchy. Recall that the star is the unique system optimum. It is well known that the Tutte graph $K_{\left\lfloor\frac{n}{2}\right\rfloor,\left\lceil\frac{n}{2}\right\rceil}$ maximizes the number of edges in a triangle free graph. Before we can state the lower bound of the price of anarchy as the ratio of the Tutte graph to the star, we need the stability of the Tutte graph. As the Tutte graph also has diameter equal to 2 , we get:

Corollary 2. The maximum bipartite graph $K_{\left\lfloor\frac{n}{2}\right\rfloor,\left\lceil\frac{n}{2}\right\rceil}$ is stable.

Corollary 3. The price of anarchy of the graph process with $n$ vertices is bounded from below by

$$
\frac{\mu\left(\left(\frac{n^{2}-n}{2 \mu}-1\right) \delta^{2}+\delta-c\right)}{(n-1)\left(\left(\frac{n}{2}-1\right) \delta^{2}+\delta-c\right)}
$$

where $\mu:=\left\lceil\frac{n}{2}\right\rceil\left\lfloor\frac{n}{2}\right\rfloor$ is the number of edges in $K_{\left\lfloor\frac{n}{2}\right\rfloor,\left\lceil\frac{n}{2}\right\rceil}$.

To show that Corollary 3 exactly states the price of anarchy for the graph process, we need to show that $K_{\left\lfloor\frac{n}{2}\right\rfloor,\left\lceil\frac{n}{2}\right\rceil}$ maximizes the number of edges among all stable graphs with $n$ vertices. That would be easy if the converse of Theorem 3 was true, i.e., all stable graphs had no triangles. This is not the case as will be shown in the next section. There, we analyze the occurrence of triangles in detail. By the end of that section we will show (Theorem 8) that all stable graphs that contain at least one triangle have less edges

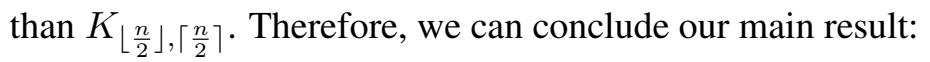

Theorem 5. For all graph processes with $n$ vertices and $c \in\left(\delta-\delta^{2}, \delta-\delta^{3}\right)$, the maximum price of anarchy equals (1) and is produced by the maximum bipartite graph $K_{\left\lfloor\frac{n}{2}\right\rfloor,\left\lceil\frac{n}{2}\right\rceil}$ against the star $K_{1, n-1}$. 

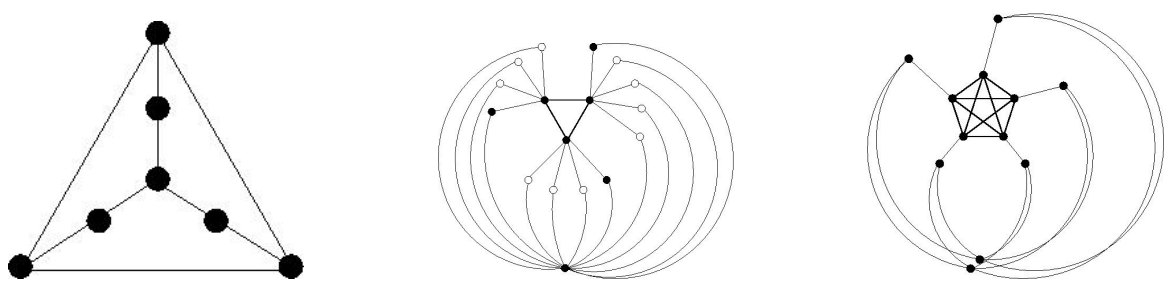

Figure 2: Stable graphs that contain a triangle.

\subsection{Triangles}

We have seen situations where a triangle is closed in the course of the graph process. There the graph process cycles and therefore does not reach a stable state. Nonetheless, there are stable graphs containing triangles.

Theorem 6. For every number of vertices greater than or equal to 7 there are graphs $G$ which are stable and contain at least one triangle.

Proof. The graphs depicted in Figure 2 are stable and contain a triangle. The black vertices in the middle graph form the left graph. The white vertices in the middle can added one by one, such that every number of vertices strictly greater than 6 is possible.

Next, we show a structural result for stable graphs with triangles that is of interest in itself.

Theorem 7. If $G$ is a stable state of the graph process and the vertex set $\{a, b, c\}$ forms a triangle in $G$, then there exists at least one $v \in V(G)$ with distances $\operatorname{dist}(a, v)=\operatorname{dist}(b, v)=\operatorname{dist}(c, v)=2$.

In order to show the statement of Theorem 7, we first need some lemmata.

Lemma 2. Let $G$ be stable and contain a triangle $\{a, b, c\}$. Then for every $i, j \in\{a, b, c\}, i \neq j$ there is a vertex in $N(i) \backslash\{a, b, c\}$ that has no edge to neither any vertex in $N(j)$ nor $j$ itself.

Proof. Assume the claim of the lemma is false. Then $j$ can reach all neighbors of $i$ via one of its own neighbors or directly. Moreover, it can reach $i$ itself in two steps via the two other arcs of the triangle. That is as good as anything $i$ can offer to $j$. Hence $j$ would drop the arc $\{i, j\}$ contradicting the stability of $G$.

Note that the premises of the following Lemmata 3 and 4 are assumptions to be falsified to prove Theorem 7. The proofs rest on the fact that, without a vertex at distance 2 to all of them, the triangle vertices behave totally jealous towards their other neighbors.

Lemma 3. Let $G$ be stable, contain a triangle $\{a, b, c\}$, and have no vertex $v$ with $\operatorname{dist}(i, v)=2$, for all $i \in\{a, b, c\}$. Then the neighborhoods of the triangle vertices form a disjoint partition of $V(G) \backslash\{a, b, c\}$.

Proof. First, it holds that $\bigcup_{i \in\{a, b, c\}} N(i)=V(G)$ because there is no vertex at distance 2 to the triangle and every stable graph has diameter less than 3. Assume vertex $v$ to be in $N(i) \cap N(j), j, i \in\{a, b, c\}, j \neq$ $i, v \notin\{a, b, c\}$. Remove the arc $\{i, v\}$. One figures out quickly that $i$ can still reach any vertex including $v$ within two steps because even in $G$ there is no vertex at distance 2 to all of the vertices $a, b, c$. Therefore, the assumption contradicts the stability of $G$.

Lemma 4. Let $G$ be stable, contain a triangle $\{a, b, c\}$, and have no vertex $v$ with $\operatorname{dist}(i, v)=2$, for all $i \in\{a, b, c\}$. Then the neighborhoods of the triangle vertices are independent sets.

Proof. Assume $v, w \in N(i) \backslash\{a, b, c\}$ for some $i \in\{a, b, c\}$ with $\{v, w\} \in E(G)$. When removing the edge $\{i, v\}$, considerations like in the previous proof show the triangle vertex $i$ to still reach every vertex within 2 steps. Hence $G$ was not stable. 
Proof of Theorem 7. Assume the claim of the theorem not to be true for $G$, containing a triangle and being stable. Let $x$ be a vertex in $N(a)$ as guaranteed to exist by Lemma 2 that has neither a connection to $b$ nor to one of its neighbors, and $y$ be a vertex in $N(b)$ that has neither a connection to $c$ nor to one of its neighbors. As $G$ is stable, its diameter is 2 . Hence, $x$ and $y$ must have a common neighbor, as they cannot be adjacent by definition of $x$. By definition of $y$, such a neighbor is neither $c$ nor one of $c$ 's neighbors. By Lemma 3 it is neither $a$ nor $b$ and by Lemma 4 it is neither a neighbor of $b$ nor of $a$. Thus, we have a contradiction.

Using Theorem 7 and the insights of Lemmata 2-4 we get the following result.

Corollary 4. Every stable graph that contains a triangle has at least 7 vertices.

We can further observe that the number of triangles in a stable graph does not need to be small. To see this consider the rightmost graph in Figure 2 where the clique in the middle can consist of $\left\lceil\frac{n-1}{2}\right\rceil$ vertices. One figures out quickly that such a graph features the biggest clique in a stable graph of $n$ vertices.

In order to determine the price of anarchy exactly, by Theorem 4 one has to look for the stable graph with the maximum number of edges. Intuitively, stable graphs with triangles should have more edges than triangle free stable graphs. But by a closer look, we see that all stable graphs with triangles have less edges than some of those without triangles, namely the $K_{\left\lfloor\frac{n}{2}\right\rfloor,\left\lceil\frac{n}{2}\right\rceil}$.

Theorem 8. For a number $n$ of vertices, the maximum bipartite graph $K_{\left\lfloor\frac{n}{2}\right\rfloor,\left\lceil\frac{n}{2}\right\rceil}$ has the maximum number of edges among all stable graphs on $n$ vertices.

Proof. We need to show that $K_{\left\lfloor\frac{n}{2}\right\rfloor,\left\lceil\frac{n}{2}\right\rceil}$ has more edges than any stable graph on $n$ vertices containing at least one triangle.

For every graph $G$ we define a random variable based on the uniform distribution over the vertices of $G$ as follows: Pick a vertex uniformly at random and sum up the degrees of its neighbors. Denote the expectation of that random variable by $\Phi(G)=\frac{1}{|V(G)|} \sum_{v \in V(G)} \sum_{u \in N(v)} d(u)$. We denote by $\mu:=$ $\left\lfloor\frac{n}{2}\right\rfloor \cdot\left\lceil\frac{n}{2}\right\rceil$ the number of edges of $K_{\left\lfloor\frac{n}{2}\right\rfloor,\left\lceil\frac{n}{2}\right\rceil}$. In order to show the statement of the theorem we prove two claims.

Claim 1. Let $G$ be a stable graph with $n$ vertices. Then $\Phi(G) \leq \Phi\left(K_{\left\lfloor\frac{n}{2}\right\rfloor,\left\lceil\frac{n}{2}\right\rceil}\right)$.

Claim 2. Let $G$ be a graph with $n$ vertices and $\mu$ edges. Then $\Phi\left(K_{\left\lfloor\frac{n}{2}\right\rfloor,\left\lceil\frac{n}{2}\right\rceil}\right) \leq \Phi(G)$.

These claims yield the statement of the theorem. Assume a stable graph $G$ on $n$ vertices with more edges than the maximum bipartite graph $K_{\left\lfloor\frac{n}{2}\right\rfloor,\left\lceil\frac{n}{2}\right\rceil}$. Arbitrarily remove edges from $G$ until the resulting graph $G^{\prime}$ has exactly as many edges as $K_{\left\lfloor\frac{n}{2}\right\rfloor,\left\lceil\frac{n}{2}\right\rceil}$. Observe that removing an edge reduces the value of $\Phi$ by definition. Hence, $\Phi\left(G^{\prime}\right)<\Phi(G)$. By the second claim $\Phi\left(K_{\left\lfloor\frac{n}{2}\right\rfloor,\left\lceil\frac{n}{2}\right\rceil}\right) \leq \Phi\left(G^{\prime}\right)$. This implies $\Phi\left(K_{\left\lfloor\frac{n}{2}\right\rfloor,\left\lceil\frac{n}{2}\right\rceil}\right)<\Phi(G)$, which contradicts the first claim. Hence there is no stable graph with more than $\mu$ edges, which proves the theorem.

It remains to prove Claims 1 and 2. In addition, we will prove an even stronger version of the first claim, namely that every stable graph containing at least one triangle has a strictly smaller value of $\Phi$ than the complete bipartite graph and consequently less edges.

Proof of Claim 1: For the maximum bipartite graph we have $\Phi\left(K_{\left\lfloor\frac{n}{2}\right\rfloor,\left\lceil\frac{n}{2}\right\rceil}\right)=\mu$. Now, consider an arbitrary, stable graph $G$. For a randomly chosen vertex $v \in V(G)$ let $N(v)$ be all neighboring vertices and $b:=$ $|N(v)|$. Partition the edges incident to the vertices in $N(v)$ into three sets: first the edges incident to $v$, second those within $N(v)$ and third the edges to other vertices. The first and the last set together contain at most $\mu$ edges, because of the bipartiteness of the subgraph formed by these edges. Every edge in the second set belongs to a triangle containing $v$. Denote the number of vertices in $N(v)$ that belong to at least one triangle with $v$ by $\ell$. Then there are at most $\frac{\ell^{2}-\ell}{2}$ edges in the second set. As $\Phi$ counts the degrees of $v$ 's neighbors, each edge in the second set counts twice. 
Assume a vertex $u$ to be part of a triangle with $v$. Why will $v$ be interested in the edge $\{u, v\}$ ? There must be at least one vertex that $v$ can reach within two steps only via $u$, or $\operatorname{dist}(u, v) \geq 3$ if the edge $\{u, v\}$ was removed. The latter is wrong, as $u$ and $v$ are in a triangle. Thus there exists a neighbor $w \neq v$ of $u$ that is not connected to any vertex in $(N(v) \cup\{v\}) \backslash\{u\}$. In other words, $u$ has an exclusive attraction to $v$ in the sense that no other vertex in $N(v) \cup\{v\}$ is connected to $w$. Therefore we have to subtract $(b-1)$ from the number of possible edges in the third set for each of the vertices in $N(v)$ that participate in a triangle with $v$. Altogether, we get that the sum over the degrees of neighbors of $v$ is at most

$$
\sum_{u \in N(v)} d(u) \leq \mu+\left(\ell^{2}-\ell\right)-\ell(b-1)
$$

if $\ell$ neighbors of $v$ participate in triangles with $v$. As $\ell \leq b$ and equation (2) holds for all vertices $v \in V(G)$, we get

$$
\Phi(G) \leq \mu .
$$

This proves Claim 1 as stated above. Moreover, a graph containing a triangle does not achieve equality in Equation (3). To show this, observe that in case of equality for each vertex the number $\ell$ must be in the set $\{0, b\}$, and if $\ell=b$ for a vertex $v$, then $N(v) \cup\{v\}$ is a clique. In other words, the neighborhood of a vertex $v$ either contains no triangle with $v$ or forms a clique together with the vertex $v$. For at least one vertex $v$ with $d(v)>1$ the latter must be true (otherwise $G$ does not contain a triangle). A neighbor $u$ of such a vertex $v$ is not interested in its edge to $v$ because $u$ has a direct edge to all of $v$ 's neighbors and can reach $v$ itself via another neighbor of $v(d(v)>1)$ within two steps. To report accurately, a graph $G$ can fulfill at most two of the following three properties which even proves the stronger version of Claim 1.

1. $G$ is stable.

2. $G$ achieves equality in Equation (3).

3. $G$ has a triangle.

Proof of Claim 2: Recall the definition of our counting function $\Phi(G)=\frac{1}{|V(G)|} \sum_{v \in V(G)} \sum_{u \in N(v)} d(u)$. Reorganizing the sums by $\sum_{v \in V(G)} \sum_{u \in N(v)} d(u)=\sum_{\{u, v\} \in E(G)} d(u)+d(v)=\sum_{v \in V(G)} d^{2}(v)$ gives:

$$
\Phi(G)=\frac{1}{|V(G)|} \sum_{v \in V(G)} d^{2}(v) .
$$

For fixed numbers of vertices and edges the expression (4) is minimized by the maximum bipartite graph. To see this consider any sequence of $n$ natural numbers $s_{i}, 1 \leq i \leq n$ with $\sum_{i} s_{i}=2 m$. We show that the degree sequence $d_{i}$ of $K_{\left\lfloor\frac{n}{2}\right\rfloor,\left\lceil\frac{n}{2}\right\rceil}$ minimizes $\sum_{i} s_{i}^{2}$ among all those sequences. Assume to the contrary that there is a sequence $s^{*}$ distinct from $d$ and minimizing the sum of squares. As it is distinct from $d$ there exists a pair $s_{i}^{*}$ and $s_{j}^{*}$ with $\left|s_{i}^{*}-s_{j}^{*}\right|>1$. W.o.l.g. we have $s_{i}^{*}>s_{j}^{*}+1$ and can look at $s^{\prime}$, where $s_{i}^{\prime}=s_{i}^{*}-1$, $s_{j}^{\prime}=s_{j}^{*}+1$ and $s_{k}^{\prime}=s_{k}^{*} \forall i \neq k \neq j$. We get $\sum_{i} s_{i}^{2}<\sum_{i} s_{i}^{* 2}$ contradicting the minimality of $s^{*}$.

\section{Further Choices of $c$ and $\delta$}

The model of Jackson, Wolinsky and Watts is rather a family of different models in their own right. The methods we used to analyze the case where $c \in\left(\delta-\delta^{2}, \delta-\delta^{3}\right)$ to a certain extend can be carried over to other cases.

Every situation gives rise to two polynomials in $\delta$ defined over the $(0,1)$ interval and mapping to the positive reals determined by the change in income of the two vertices in question. We can also interpret the set $(0,1) \times \mathbb{R}^{+}$in which the graphs (here: graphs of functions) of those polynomials live as the set of all 


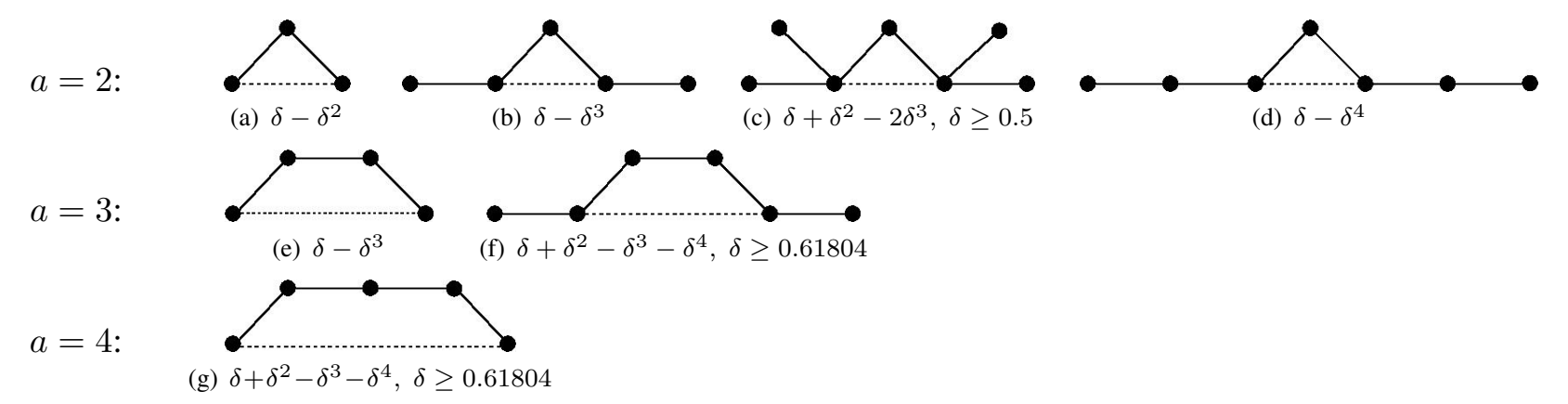

Figure 3: Subnetworks that need to be considered in the proof of Theorem 9. The caption determines the benefit of the dashed edge. In the considered case, only edges having a benefit of strictly more than $\delta-\delta^{4}$ will be inserted during the graph process. The lower bounds on $\delta$ in Figures 3(f) and 3(g) are needed if we consider the graph process starting from an empty graph. These bounds guarantee that $c<\delta$ which makes the insertion of edges into an empty graph possible.

graph processes for a fixed number of vertices $n$, because they are defined by a $\delta$ coordinate in $(0,1)$ and a $c$ coordinate in $\mathbb{R}^{+}$. In this picture a decision is positive for exactly those graph processes that are below the polynomials of both end-vertices. This visualizes how the polynomials separate the set of all processes in those for which they are positive and those for which they are negative.

Restricting to $c \in\left(\delta-\delta^{2}, \delta-\delta^{3}\right)$ we exploit the nature of $\delta-\delta^{2}$ and $\delta-\delta^{3}$ as threshold functions. We call a function $f:(0,1) \rightarrow \mathbb{R}^{+}$a threshold function if for every polynomial $p$ that stems from a situation we have $\exists x_{0} \in(0,1): p\left(x_{0}\right)<f\left(x_{0}\right) \Rightarrow p(x)<f(x) \forall x \in(0,1)$ and $\exists x_{0} \in(0,1): p\left(x_{0}\right)>f\left(x_{0}\right) \Rightarrow$ $p(x)>f(x) \forall x \in(0,1)$. One may carry on looking for threshold functions and redo our analyze for these cases. The next theorem gives the next threshold function.

Theorem 9. For fixed $n$ all graph process with $c \in\left(\delta-\delta^{3}, \delta-\delta^{4}\right)$ are identical.

Proof. We have to show, that a situation is either positive for all graph processes in the interval or for none.

Classify all situations by the distance $a:=\operatorname{dist}(u, v)$ of the considered pair $\{u, v\}$ of vertices. Figure 3 gives the decisive situations for each choice of $a$. Under the premise of the theorem all situations with $a \geq 4$ will yield a positive decision: The income for $u$ grows at least from the part of $v$ and $v$ 's direct neighbor on a shortest path to $u$. Their increase in income sums to $\delta-\delta^{4}+\delta^{2}-\delta^{3}>\delta-\delta^{4}$ which is greater than $c$.

For $a=3$ the end-vertices $u$ and $v$ must attract each other with vertices not located on a shortest path between $u$ and $v$ to have a gain greater than $c>\delta-\delta^{3}$. Therefore they at least have an increase of $\delta-\delta^{3}+\delta^{2}+\delta^{3}$ which is sufficient for all graph processes.

It remains to consider situations where $a=2$. Here at least two further vertices outside the shortest paths are required for the situation to be positive for at least one process of the interval. These can yield either the situation depicted in Figure 3(d) or Figure 3(c). All other situations that are decided positive for a $c \in\left(\delta-\delta^{3}, \delta-\delta^{4}\right)$ contain one of these as subgraphs. As the increase in income for situation in Figure 3(d) is the smaller one. This completes the theorem.

One may suppose that all polynomials stemming from situations are threshold functions. But this is not the case, as there are situations yielding polynomials that intersect each other in the open interval $(0,1)$.

Concider the two situations depicted in Figure 4. In both cases the black vertex has so many neighbors that the opposite end-vertex of the dashed edge will endorse the insertion of the dashed edge. The polynomial for the black vertex in the left situation minus that of the black vertex to the right gives: $\delta^{3}-\delta^{5}-\left(\delta^{4}-\delta^{7}\right)$. For some values of $\delta \in(0,1)$ this is negative for some it is positive. In other words, some processes will insert the edge in the left situation but not in the right situation, whereas other processes will do the contrary 

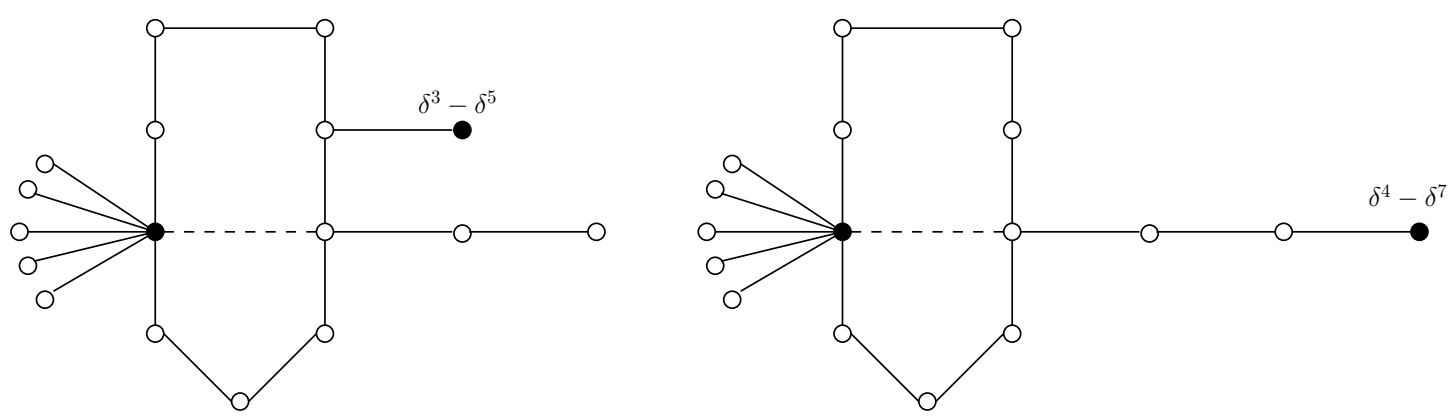

Figure 4: The insertion of the edge in question (dashed) strongly depends on the actual choice of $c$ and $\delta$.

in both situations. This implies that for predicting the process' behavior it is no longer sufficient to specify one parameter by bounds of the other parameter.

Nevertheless, general results, e.g., concerning the price of anarchy, might be achievable along the lines of this work. Choose a graph process, where the star is optimal. Every stable graph will have diameter less than $k$ if $c<\delta-\delta^{k-1}$. What can be said about graphs that have the maximum number of edges among all those containing no cycle smaller than $k-1$, i.e., in graph theoretic terms, which have girth $k-1$ ? They are not necessarily stable, but none of their present edges will currently be removed.

Recall our central result, namely that $K_{\left\lfloor\frac{n}{2}\right\rfloor,\left\lceil\frac{n}{2}\right\rceil}$ has more edge than stable graphs with triangles. The $K_{\left\lfloor\frac{n}{2}\right\rfloor,\left\lceil\frac{n}{2}\right\rceil}$ is an extremal graph in the sense that it maximizes the number of edges for girth 4 .

We conjecture that in general the graphs with maximum number of edges for girth $k-1$ are a good approximation for those maximizing the price of anarchy. The maximum number of edges in a graph of fixed girth is a long standing question in extremal graph theory (cf. $[2,8,10]$ ). Most astonishingly the known upper bounds for this number of edges would imply that the price of anarchy becomes constant. For simplicity account for the price of anarchy as the greatest fraction between the number of edges in a stable and in an optimal graph. If our conjecture holds that the maximum graph of girth $k-1$ approximates the price of anarchy for graph processes with $\delta-\delta^{k-1}<c<\delta-\delta^{k}$, this means that this price of anarchy would drop from worst $(\mathcal{O}(n))$ to best $(\mathcal{O}(1))$ as $k$ growth. In other words, the users form an optimal network if the costs are very low $\left(c<\delta-\delta^{2}\right)$ and an almost optimal network if the costs are very high. The worst outcome is then caused by costs $c \in\left(\delta-\delta^{2}, \delta-\delta^{3}\right)$, which is the case we have analyzed.

\section{References}

[1] S. Albers, S. Eilts, E. Even-Dar, Y. Mansour, and L. Roditty. On Nash equilibria for a network creation game. In Proceedings of the 17th ACM-SIAM Symposium on Discrete Algorithms (SODA'06), pages 89-98, 2006.

[2] N. Alon, S. Hoory, and N. Linial. The Moore bound for irregular graphs. Graphs and Combinatorics, 18(1):5357, 2002.

[3] E. Anshelevich, A. Dasgupta, J. Kleinberg, E. Tardos, T. Wexler, and T. Roughgarden. The price of stability for network design with fair cost alloccation. In Proceedings of the 45th Annual IEEE Symposium on Foundations of Computer Science (FOCS'04), pages 295-304, 2004.

[4] E. Anshelevich, A. Dasgupta, E. Tardos, and T. Wexler. Near-optimal network design with selfish agents. In Proceedings of 35th Annual ACM Symposium on Theory of Computing (STOC'03), pages 511-520, 2003.

[5] V. Bala and S. Goyal. A non-cooperative model of network formation. Journal of Econometrics, 68(5):1181$1229,2000$.

[6] A.-L. Barabási and R. Albert. Emergence of scaling in random networks. Science, 286:509-512, 1999. 
[7] J. Corbo and D. C. Parkes. The price of selfish behavior in bilateral network formation. In Proceedings of the 24th ACM Symposium on Principles of Distributed Computing (PODC'05), 2005.

[8] R. D. Dutton and R. C. Brigham. Edges in graphs with large girth. Graphs and Combinatorics, 7:315-321, 1991.

[9] A. Fabrikant, A. Luthra, E. Maneva C. H. Papadimitriou, and S. Shenker. On a network creation game. In Proceedings of the 22nd annual Symposium On Principles of Distributed Computing (PODC'03), pages 347351, 2003.

[10] S. Hoory. The size of bipartite graphs with a given girth. Journal of Combinatorial Theory. Series B, 86(2):215220, 2002.

[11] M. O. Jackson. A survey of models of network formation: Stability and efficiency. In G. Demange and M. Wooders, editors, Group Formation in Economics: Networks, Clubs and Coalitions, chapter 1, pages 11-57. Cambridge University Press, Cambridge, UK, 2004.

[12] M. O. Jackson and A. Wolinsky. A strategic model of social and economic networks. Journal of Economic Theory, 71:44-74, 1996. reprinted in Networks and Groups: Models of Strategic Formation, edited by Dutta and Jackson, Springer-Verlag, Heidelberg 2003.

[13] E. Koutsoupias and C. H. Papadimitriou. Worst-case equilibria. In Proceedings of the 16th STACS, pages 404413, 1999.

[14] M. A. Melendez-Jiminez. Network formation and coordination: Bargaining the division of link costs. Presented at 57th European Meeting of the Econometric Society, August 2002. http://www.webdeptos.uma.es/THEconomica.net1.pdf.

[15] J. F. Nash. Non-cooperative games. Annals of Mathematics, 54(286-295), 2951.

[16] A. Watts. A dynamic model of network formation. Games and Economic Behavior, 34:331-341, 2001. 
Reports from the group

\title{
“Combinatorial Optimization and Graph Algorithms"
}

\author{
of the Department of Mathematics, TU Berlin
}

2007/19 Nadine Baumann and Sebastian Stiller: The Price of Anarchy of a Network Creation Game with Exponential Payoff

2006/32 Romeo Rizzi and Christian Liebchen: A New Bound on the Length of Minimum Cycle Bases

2006/24 Christian Liebchen and Sebastian Stiller: Delay Resistant Timetabling

2006/08 Nicole Megow and Tjark Vredeveld: Approximation Results for Preemptive Stochastic Online Scheduling

2006/07 Ekkehard Köhler and Christian Liebchen and Romeo Rizzi and Gregor Wünsch: Reducing the Optimality Gap of Strictly Fundamental Cycle Bases in Planar Grids

2006/05 Georg Baier and Thomas Erlebach and Alexander Hall and Ekkehard Köhler and Heiko Schilling: LengthBounded Cuts and Flows

2005/30 Ronald Koch and Martin Skutella and Ines Spenke: Maximum k-Splittable Flows

2005/29 Ronald Koch and Ines Spenke : Complexity and Approximability of k-Splittable Flows

2005/28 Stefan Heinz and Sven O. Krumke and Nicole Megow and Jörg Rambau and Andreas Tuchscherer and Tjark Vredeveld: The Online Target Date Assignment Problem

2005/18 Christian Liebchen and Romeo Rizzi: Classes of Cycle Bases

2005/11 Rolf H. Möhring and Heiko Schilling and Birk Schütz and Dorothea Wagner and Thomas Willhalm: Partitioning Graphs to Speed Up Dijkstra's Algorithm.

2005/07 Gabriele Di Stefano and Stefan Krause and Marco E. Lübbecke and Uwe T.Zimmermann: On Minimum Monotone and Unimodal Partitions of Permutations

2005/06 Christian Liebchen: A Cut-based Heuristic to Produce Almost Feasible Periodic Railway Timetables

2005/03 Nicole Megow, Marc Uetz, and Tjark Vredeveld: Models and Algorithms for Stochastic Online Scheduling

2004/37 Laura Heinrich-Litan and Marco E. Lübbecke: Rectangle Covers Revisited Computationally

2004/35 Alex Hall and Heiko Schilling: Flows over Time: Towards a more Realistic and Computationally Tractable Model

2004/31 Christian Liebchen and Romeo Rizzi: A Greedy Approach to Compute a Minimum Cycle Bases of a Directed Graph

2004/27 Ekkehard Köhler and Rolf H. Möhring and Gregor Wünsch: Minimizing Total Delay in Fixed-Time Controlled Traffic Networks

2004/26 Rolf H. Möhring and Ekkehard Köhler and Ewgenij Gawrilow and Björn Stenzel: Conflict-free Real-time AGV Routing

2004/21 Christian Liebchen and Mark Proksch and Frank H. Wagner: Performance of Algorithms for Periodic Timetable Optimization

2004/20 Christian Liebchen and Rolf H. Möhring: The Modeling Power of the Periodic Event Scheduling Problem: Railway Timetables — and Beyond

2004/19 Ronald Koch and Ines Spenke: Complexity and Approximability of k-splittable flow problems 
2004/18 Nicole Megow, Marc Uetz, and Tjark Vredeveld: Stochastic Online Scheduling on Parallel Machines

2004/09 Marco E. Lübbecke and Uwe T. Zimmermann: Shunting Minimal Rail Car Allocation

2004/08 Marco E. Lübbecke and Jacques Desrosiers: Selected Topics in Column Generation

2003/050 Berit Johannes: On the Complexity of Scheduling Unit-Time Jobs with OR-Precedence Constraints

2003/49 Christian Liebchen and Rolf H. Möhring: Information on MIPLIB's timetab-instances

2003/48 Jacques Desrosiers and Marco E. Lübbecke: A Primer in Column Generation

2003/47 Thomas Erlebach, Vanessa Kääb, and Rolf H. Möhring: Scheduling AND/OR-Networks on Identical Parallel Machines

2003/43 Michael R. Bussieck, Thomas Lindner, and Marco E. Lübbecke: A Fast Algorithm for Near Cost Optimal Line Plans

2003/42 Marco E. Lübbecke: Dual Variable Based Fathoming in Dynamic Programs for Column Generation

2003/37 Sándor P. Fekete, Marco E. Lübbecke, and Henk Meijer: Minimizing the Stabbing Number of Matchings, Trees, and Triangulations

2003/25 Daniel Villeneuve, Jacques Desrosiers, Marco E. Lübbecke, and François Soumis: On Compact Formulations for Integer Programs Solved by Column Generation

2003/24 Alex Hall, Katharina Langkau, and Martin Skutella: An FPTAS for Quickest Multicommodity Flows with Inflow-Dependent Transit Times

2003/23 Sven O. Krumke, Nicole Megow, and Tjark Vredeveld: How to Whack Moles

2003/22 Nicole Megow and Andreas S. Schulz: Scheduling to Minimize Average Completion Time Revisited: Deterministic On-Line Algorithms

2003/16 Christian Liebchen: Symmetry for Periodic Railway Timetables

2003/12 Christian Liebchen: Finding Short Integral Cycle Bases for Cyclic Timetabling

762/2002 Ekkehard Köhler and Katharina Langkau and Martin Skutella: Time-Expanded Graphs for FlowDependent Transit Times

761/2002 Christian Liebchen and Leon Peeters: On Cyclic Timetabling and Cycles in Graphs

752/2002 Ekkehard Köhler and Rolf H. Möhring and Martin Skutella: Traffic Networks and Flows Over Time

739/2002 Georg Baier and Ekkehard Köhler and Martin Skutella: On the $k$-splittable Flow Problem

736/2002 Christian Liebchen and Rolf H. Möhring: A Case Study in Periodic Timetabling

Reports may be requested from: $\quad$ Sekretariat MA 6-1

Fakultät II - Institut für Mathematik

TU Berlin

Straße des 17. Juni 136

D-10623 Berlin - Germany

e-mail: klink@math.TU-Berlin.DE

Reports are also available in various formats from

http: //www.math.tu-berlin.de/coga/publications/techreports/

and via anonymous ftp as

ftp: //ftp.math.tu-berlin.de/pub/Preprints/combi/Report-number-year.ps 